\title{
Directional hypokinesia in spatial hemineglect: a case study
}

\author{
G Bottini, R Sterzi, G Vallar
}

\begin{abstract}
A patient with an ischaemic lesion involving the right frontal lobe and basal ganglia showed left spatial hemineglect in visuomotor exploratory tasks, requiring the use of the right unaffected hand. Her performance was, however, entirely preserved, with no evidence of neglect, when she was required to identify targets among distractors in both the left and right halves of space, and in the Wundt-Jastrow illusion test. The latter tasks do not require any arm movement in extrapersonal space. In this patient spatial hemineglect may be explained in terms of defective organisation of movements towards the left half-space (directional hypokinesia). The frontal lesion of the patient may be the neural correlate of this selective disorder. This pattern of impairment may be contrasted with the typical deficit found in patients with right brain damage with perceptual neglect. One case had a defective performance both in visuomotor and in purely perceptual tasks.
\end{abstract}

Patients with spatial hemineglect typically fail to explore the half-space contralateral to the side of the cerebral lesion. This exploratory deficit may be due to their inability to plan and initiate movements towards the neglected halfspace, even when the unaffected ipsilateral hand is used. This premotor deficit (directional hypokinesia) may be distinguished from the perceptual aspects of spatial hemineglect. ${ }^{1-3}$ We report a patient with right brain damage with a frontal lesion, who showed a dissociation between hypokinetic and perceptual aspects of spatial hemineglect. This pattern of performance is contrasted with the behaviour of a second patient with a temporo-parietal lesion and a perceptual visuo-spatial neglect.

Divisione di

Neurologia, Ospedale di Niguarda, Milan

G Bottini

R Sterzi

Istituto di Clinica

Neurologica,

Universitad di Milano,

Milan, Italy

G Vallar

Correspondence to:

Dr Vallar, Istituto di Clinic Neurologica, Università di

Milano, Via F Sforza 35,

20122, Milan, Italy

Received 18 March 1991

and in revised form

12 September 1991 .

Accepted 28 October 1991 head and eyes. Neurological examination revealed a left hypotonic hemiplegia with enhanced left tendon reflexes and a left Babinski sign, and a severe left hypoaesthesia for touch, pinprick and proprioceptive stimulations. On confrontation, the patient could accurately detect single left- and right-sided visual stimuli, but showed left extinction on bilateral simultaneous stimulation. The patient was aware of her motor deficits and did not show personal neglect for her left limbs. Blood pressure was $130 / 180 \mathrm{~mm} \mathrm{Hg}$, pulse $80 / \mathrm{min}$, and temperature $36^{\circ} \mathrm{C}$. Respiration was regular at a normal rate. Routine blood and urine examination gave normal results. ECG, chest $x$-ray and perfusion lung scan confirmed pulmonary embolism. A CT performed a week after stroke onset, showed a right corticosubcortical hypodense area involving the dorso-lateral frontal regions and the basal ganglia, and extending posteriorly into the anterior parietal cortex. Two weeks later a second CT revealed haemorrhagic infarction (fig 1).

\section{Neuropsychological assessment}

Baseline tests. The patient was examined two weeks after the onset of stroke. She had a left hemiplegia, hypoaesthesia and visual extinction, while she recovered from the left-sided conjugate gaze paresis. The patient showed a left visuo-spatial neglect in a number of exploratory tasks, in which a sheet of paper was located in front of her, with the centre of the sheet on the midsagittal plane of her trunk. For these tasks she received instructions to search and cross out targets (interspersed among distractors) located in the left and in the right halves of a sheet using her right unaffected hand. In the Bells Test ${ }^{4}$ the target stimuli were 35 black outlines of bells ( 17 left-sided, one central, 17 right-sided). Each bell was $7 \mathrm{~mm}$ high and $5 \mathrm{~mm}$ wide. The 280 distractors were outlines of common objects (such as, a fish, a house, a horse) comparable in size to the bells. The targets and the distractors, printed on a $29 \times 21 \mathrm{~cm}$ sheet, were distributed in seven columns; each column included five targets and 40 distractors. The patient crossed out only one out of 17 left sided targets (bells), while her performance was errorless in the right half of the sheet (17 out of 17). In the Letter Cancellation Task 312 letters $4 \mathrm{~mm}$ high (104 target letters " $\mathrm{H}$ " and 208 distractor letters) were printed in six rows on a $41.4 \times 29.5 \mathrm{~cm}$ sheet. The patient crossed out only $35 / 104$ " $H$ " target letters. Her omissions included all 51 left sided targets and 18 " $\mathrm{H}$ " letters located in the left portion of the right 
Figure $C T$ showing a cortico-subcortical lesion of the right pre-motor and sensory-motor cortex, the superior and middle parts of the temporal lobe, marginally involving the posterior parietal regions. The right periventricular white matter, the lenticular and the caudate nuclei, the anterior limb of the internal capsule and other structures (external capsule, claustrum, insula) are also involved.
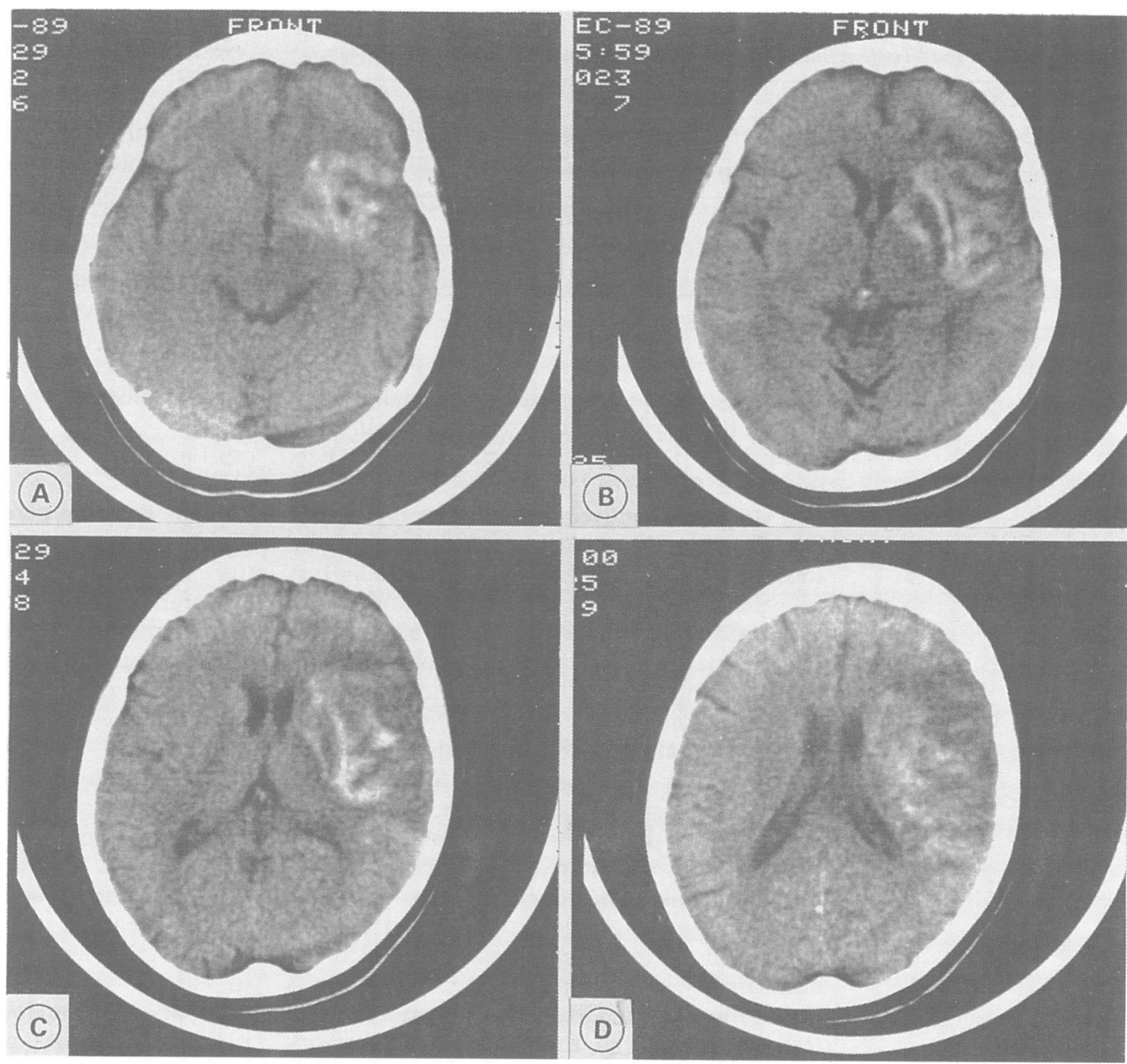

half of the sheet. During the execution of this task we noted that she looked at the left half of the sheet, saying that she saw a great deal of 'Hs" on the left and that she was well able to identify them among other letters. The patient was nevertheless unable to cross out such leftsided "Hs", or even to point to them with her right hand. This clinical observation raised the possibility that her left neglect was mainly due to directional hypokinesia, and therefore confined to tasks requiring the motor exploration by the unaffected arm of the half-space contralateral to the lesion.

Crossing Out vs identification of left-sided stimuli Prompted by this clinical observation, we devised two conditions of the Bells Test ${ }^{4}$ and of the Letter Cancellation Test, ${ }^{5}$ to explore the role of directional hypokinesia in producing left hemineglect in the patient. On the Bells Test ${ }^{4}$ sheet six bells were added, one stimulus in each column, with the exception of the central one. This version of the task sheet therefore comprised 40 target bells, 20 right sided and 20 left sided. The sheet of the Letter Cancellation Test was identical to the original version. ${ }^{5}$ In the Crossing Out condition the patient received instructions to cross out with a pencil the stimulus (a "bell" or an " $H$ ") indicated by the examiner using a pointer, abstaining from crossing out stimuli different from the target. As in the previous tasks she used the right unaffected hand. In the Identification condition the patient's task was to say whether or not the stimulus indicated by the examiner was a "bell" or an " $H$ ". In both conditions the examiner (GB) sat in front of the patient. Before pointing to each stimulus, the examiner verbally warned the patient, without mentioning whether the indicated stimulus would have been left or right sided. In both conditions the centre of the sheet was located on the midsagittal plane of the patient's trunk. Two blocks of trials were given for each condition, using an ABBA design. The crossing out condition was tested first. In the Bells Test each block comprised 40 stimuli (20 left sided and 20 right sided, 20 bells and 20 different objects) in a random fixed order. In the Letter Cancellation Test, each block included 104 stimuli ( 52 left sided and 52 right sided, 52 " $\mathrm{H}$ " and 52 different letters) in a random fixed order.

In the Identification condition the patient was fully accurate in discriminating target and non-target stimuli: even though she had received instructions to say whether each item pointed out by the examiner was or was not the target, she also spontaneously named each stimulus, with complete accuracy. In the Crossing Out condition the patient accurately complied with the directions and never crossed 
Table 1 Case 1. Number of left sided correct responses (percentages in brackets) in the Identification and Crossing Out conditions of the Bells and Letter Tests. The scores refer to target ("Bells" and " $\mathrm{Hs}$ ") stimuli

\begin{tabular}{|c|c|c|c|c|c|c|}
\hline & \multicolumn{6}{|c|}{ Condition } \\
\hline & \multicolumn{3}{|c|}{ Identification } & \multicolumn{3}{|c|}{ Crossing out } \\
\hline & Block 1 & Block 2 & Total & Block 1 & Block 2 & Total \\
\hline Bells & $10 / 10$ & $10 / 10$ & $\begin{array}{l}20 / 20 \\
(100 \%)\end{array}$ & $4 / 10$ & $6 / 10$ & $\begin{array}{l}10 / 20 \\
(50 \%)\end{array}$ \\
\hline Letter & $26 / 26$ & $26 / 26$ & $\begin{array}{l}52 / 52 \\
(100 \%)\end{array}$ & $22 / 26$ & $18 / 26$ & $\begin{array}{l}40 / 52 \\
(77 \%)\end{array}$ \\
\hline
\end{tabular}

out non-target stimuli. For. the right sided stimuli her performance was errorless in both conditions. Table 1 shows the patient's performance for left sided targets: it is apparent that she was selectively impaired in the Crossing Out condition as compared with the Identification condition, both in the Bells Test $\left(\chi^{2}=13.33, \mathrm{df}=1, \mathrm{p}<0.001\right)$ and in the Letter Cancellation Test $\left(\chi^{2}=13 \cdot 57, \mathrm{df}=1\right.$, $\mathrm{p}<0.001)$. The dissociation between defective Crossing Out and preserved identification was clearly illustrated by the patient's behaviour when making a left sided omission error in the Crossing Out condition. She correctly named the target but did not initiate any movement or the motor action was terminated shortly after the start, before crossing the midline. This behaviour contrasts with her errorless Crossing Out of right sided stimuli. On specific questioning the patient denied any abnormality in her performance. The patient's impairment in the Crossing Out condition was less severe than in the standard exploratory tasks reported above. In the Crossing Out condition she was, however, given a number of cues (each stimulus was indicated by the examiner after a verbal warning). These are lacking in the standard versions of the tasks, ${ }^{45}$ in which patients are simply required to search for targets among distractors, without any further prompting.

Wundt-Fastrow Illusion Test

These findings suggest that the patient's spatial hemineglect may be traced back to her inability to initiate and execute movements towards the left half-space. To gather additional evidence corroborating this interpretation our patient was given the Wundt-Jastrow Illusion Test devised by Massironi et al ${ }^{6}$ to detect spatial hemineglect. In this test each stimulus comprises two black fans, identical in area but different in shape, whereby one appears longer than the other, even though they are equal in length. In the version by Massironi et al the illusory effect is produced either by the left or by the right extremities of the fans. Patients with left spatial hemineglect make errors (that is, they fail to show the normal illusory effect) when the illusion arises in the left halves of the fans, while their performance is normal (that is, they show the illusory effect) when the illusion is produced by the right halves of the fans. The test requires only a perceptual judgement upon the stimulus, without any motor exploration of the extrapersonal space. Defective performance in the Wundt-Jastrow
Illusion Test may therefore be taken as an indication that perceptual neglect is present. The patient was not expected to be defective in this task, as the previous studies had shown neglect only when the paradigm required the motor exploration of the left extrapersonal space, contralateral to the lesion. The patient was instructed to say whether the upper or the lower fan was longer. Forty stimuli (20 with a right sided and 20 with a left sided illusory effect) were given in a random fixed order. The patient's performance was errorless, scoring $\mathbf{4 0}$ out of 40 correct answers, with a normal illusory effect.

\section{CONTROL PATIENT}

Case 2

Patients showing perceptual left neglect in the Wundt-Jastrow Illusion Test are typically defective also in visuomotor tasks requiring the exploration of the left half-space. ${ }^{67}$ This suggests that a defective perceptual representation of the extrapersonal space contralateral to the lesion may produce neglect with both verbal and motor responses. Patients with perceptual neglect may therefore show a comparable impairment in the identification and Crossing Out conditions of the present task. We assessed this prediction in a patient with right brain damage with a severe perceptual neglect, as assessed by the Wundt-Jastrow Illusion Test.

An 80 year old right handed woman with five years of schooling was admitted to hospital due to sudden onset of left hemiparesis. She had not had any previous psychiatric or neurological disease. She was alert and cooperative, with a steady rightward deviation of head and gaze. A neurological examination revealed a left hemiparesis, more severe in the upper limb, and a left Babinski sign. No somatosensory deficits for touch, pinprick and proprioceptive stimulations were found. On confrontation, a left homonymous hemianopia was shown. The patient denied her motor and visual field deficits. Blood pressure was $160 / 80$ $\mathrm{mm} \mathrm{Hg}$, pulse $80 / \mathrm{min}$, temperature $36 \cdot 8^{\circ} \mathrm{C}$. Routine blood and urine examination, chest $x$ ray and ECG were normal. CT performed one week after the onset of the neurological deficit showed a tumour which involved the right subcortical temporo-parietal region, compressed the right lateral ventricle and was surrounded by oedema. Intravenous injection of contrast medium produced a homogeneous enhancement in the tumour mass.

\section{Neuropsychological assessment}

The patient was examined 10 days after the onset of the neurological deficits. She had a left hemiparesis and a left homonymous hemianopia, and had recovered from the left sided conjugate gaze paresis. The patient showed a severe perceptual neglect on the WundtJastrow Illusion Test. The patient gave only one out of 20 correct answers when the illusion was left sided. By contrast, her performance was virtually errorless (19 out of 20 correct responses) when the illusion was right sided. In the Bells Test ${ }^{4}$ she failed to cross out all 17 left sided targets and 12 out of 17 right sided 
Table 2 Case 2-control patient. For conditions and scores see table 1.

\begin{tabular}{|c|c|c|c|c|c|c|}
\hline & \multicolumn{6}{|c|}{ Condition } \\
\hline & \multicolumn{3}{|c|}{ Identification } & \multicolumn{3}{|c|}{ Crossing out } \\
\hline & Block 1 & Block 2 & Total & Block 1 & Block 2 & Total \\
\hline Bells & $5 / 10$ & $5 / 10$ & $\begin{array}{l}10 / 20 \\
(50 \%)\end{array}$ & $6 / 10$ & $5 / 10$ & $\begin{array}{l}11 / 20 \\
(55 \%)\end{array}$ \\
\hline Letter & $16 / 26$ & $17 / 26$ & $\begin{array}{l}33 / 52 \\
(63 \%)\end{array}$ & $15 / 26$ & $16 / 26$ & $\begin{array}{l}31 / 52 \\
(60 \%)\end{array}$ \\
\hline
\end{tabular}

targets; these right sided omissions involved the left part of the right half of the sheet. She showed a similar pattern of impairment in the Letter Cancellation Task, ${ }^{5}$ in which she failed to cross out all 51 left sided letters and 32 out of 53 right sided letters. The patient's performance in the Identification and Crossing Out conditions of the Bells and Letter Tests is shown in table 2. The patient's errors were confined to the left half of the sheet; in the case of right sided targets her performance was entirely correct. Unlike case 1 she showed a comparable impairment in the two conditions of the task. As previously found in case 1, the overall performance of our control patient was better in the Identification and Crossing Out conditions, compared with the standard versions of the tasks. ${ }^{45}$

\section{Discussion}

Case 1 shows a dissociation in the manifestations of left visuospatial hemineglect. A defective exploration of extrapersonal space is present only when the task requires the execution of movements in the left half-space, by making use of the right unaffected arm. The patient is entirely unimpaired in visuospatial tasks, such as the identification of targets and the Wundt-Jastrow Illusion Test, which involve only a perceptual analysis of the left half-space. By contrast, the control patient, case 2 , who had a severe perceptual neglect, assessed by the Wundt-Jastrow Illusion Test, has a defective performance in all exploratory tasks, without any dissociation between motor $v s$ identification response modalities. In the present experimental paradigm, a disordered perceptual representation of the left extrapersonal space may affect the programming of all types of responses to left sided targets, both when output is a lateralised motor response, and when the identification of the stimulus is required.

The selective impairment of case 1 in the execution of arm movements towards the left half-space can be explained in terms of neither primary motor deficits nor motor neglect. ${ }^{8}$ She used her right unaffected arm and her per- formance was errorless when the target was located in the right half-space. The failure to plan and initiate movements towards the halfspace contralateral to the lesion is a component of spatial hemineglect. ${ }^{1-3}$ In most published cases both perceptual and premotor deficits contribute to producing spatial hemineglect, ${ }^{3}$ while in a few patients directional hypokinesia has been shown to be the main factor. ${ }^{2}$ In case 1 the exploratory deficit can be entirely attributed to directional hypokinesia, as her performance towards the left half-space is entirely preserved when arm movements are not required. The selectivity of the patient's behavioural impairment has an anatomical counterpart. The CT showed a lesion involving the right frontal lobe and basal ganglia, with comparatively minor damage of the posterior parietal regions. Conversely, the control patient (case 2), who showed a perceptual neglect with no differences related to output modality, had a temporo-parietal lesion. Suggestions have been made, ${ }^{910}$ and some empirical evidence exists in humans, ${ }^{2311}$ that directional hypokinesia may be produced by anterior cortical and subcortical lesions, while the perceptual aspects of neglect are more frequently associated with posterior (parietal) lesions. In the present case, the anterior localisation of the ischaemic lesion assessed by CT may account for the unusually clear-cut dissociation between premotor and perceptual aspects of neglect.

This study was supported in part by CNR and MPI grants to GV.

1 Watson RT, Miller BD, Heilman KM. Nonsensory neglect. Ann Neurol 1978;3:505-8.

2 Coslett HB, Bowers D, Fitzpatrick E, Haws B, Heilman KM. Directional hypokinesia and hemispatial inattention KM. Directional hypokinesia and hem

3 Bisiach E, Geminiani G, Berti A, Rusconi ML. Perceptual and premotor factors of unilateral neglect. Neurology 1990;40:1278-81.

4 Gauthier L, Dehaut F, Joanette Y. The bells test: a quantitative and qualitative test for visual neglect. Int $\mathfrak{f}$ Clin Neuropsychol 1989;11:49-54.

5 Diller $\mathrm{L}$, Weinberg J. Hemi-Inattention in rehabilitation. The evolution of a rational remediation program. In: Weinstein EA, Friedland RP, eds. Hemi-inattention and hemisphere EA, Friedland RP, eds. Hemi-inattention and hemisphere Raven Press, 1977:62-82.

6 Massironi M, Antonucci G, Pizzamiglio L, Vitale MV, Zoccolotti P. The Wundt-Jastrow illusion in the study of Zoccolotti P. The Wundt-Jastrow illusion in the study of
spatial hemi-inattention. Neuropsychologia 1988;26: spatial

7 Pizzamiglio L, Cappa S, Vallar G, Zoccolotti P, Bottini G, Ciurli $P$, et al. Visual neglect for far and near extrapersonal space in humans. Cortex 1989;25:471-7.

8 Critchley M. The parietal lobes. New York: Hafner Press, 1953.

9 Heilman KM, Watson RT, Valenstein E. Neglect and related disorders. In: Heilman KM, Valenstein E, eds. Clinical Neuropsychology, 2nd ed. Oxford: Oxford University Press, 1985:243-93.

10 Mesulam M-M. A cortical network for directed attention and unilateral neglect. Ann Neurol 1981;11:309-25.

11 Tegnér $R$, Levander $M$. Through a looking glass. A new technique to demonstrate directional hypokinesia in unilateral neglect. Brain 1991;114:1943-5. 